\title{
Efectos del uso de la Tecnología Digital en el cerebro y en el psique. Análisis de las evidencias y dos propuestas.
}

\author{
Bricolo, F.*; Serpelloni, G.** \\ *Médico, Psiquiatra, Psicoterapeuta. * ${ }^{*}$ Médico, Director de Departamento.
}

Enviar correspondencia a:

Departamento de Dependencias ·Via Germania 20 l-37135-Verona (Italia)·fbricolo@dronet.org

\section{RESUMEN}

En la segunda mitad de los años 90 se publicaron algunos artículos sobre un "nuevo problema" que los medios de comunicación bautizaron con las siglas IAD, de Internet Addiction Disorders (Trastornos de la Adicción a Internet) Estas siglas han sido utilizadas con gran éxito por los medios de comunicación, en cambio, en los estudios científicos sólo han tenido un valor anecdótico. Objetivo. En este artículo, los autores presentan una primera propuesta de definición, de cinco puntos, de este "nuevo problema", un examen de las evidencias acerca de éste y seis criterios que la investigación debería seguir en el futuro. Método. Primero: Se propone una definición de cinco puntos de este "nuevo problema". Segundo: Se busca en la base de datos on-line las evidencias que estudian este "nuevo problema". Tercero: los autores han elegido algunas variables (tecnología, problema, metodología) con las que se interpretan las evidencias halladas y cada evidencia se analiza con estas tres clases de variables. Resultado. El primer punto es que es posible identificar, en una segunda generación de estudios sobre la asociación/correlación entre UTD (Usuarios de las Tecnologías Digitales) y sus efectos en el cerebro y en la psique, como enfermedad mental. Estos estudios investigan cohortes de UTD, utilizando instrumentos Golden Standard (estandarizados y validados) y éste debería ser un asunto incuestionable. EI segundo punto es que, aunque la población de UTD sea pequeña y dishomogénea, existen evidencias de enfermedad DSM-IV Ejes I y II. Acerca del problema de la dependencia/adicción hay que tener en cuenta que,. por el momento, con el DSM-IV/ICD10 es posible hacer un diagnostico de "dependencia" sólo con "sustancias". Hay algunas evidencias anecdóticas que muestran un problema de "dependencia/adicción" en los UTD. Consideraciones. Los autores proponen seis criterios con los que han de efectuarse los futuros estudios. Es realmente necesario organizar los estudios a nivel internacional siguiendo los seis criterios propuestos

Palabras clave: Internet, adicción, definición, criterios, DSM-IV

\section{ABSTRACT}

In the second part of the years ' 90 were published some articles about a "new problem" that the mass media have named IAD acronyms of Internet Addiction Disorders. This acronyms used by mass media where unfortunately have met success instead of the scientific studies have anecdotic. The aim. In this article the AA present a first proposal of a five-point definition of this "new problem", a review of the evidences about this problem and six criteria that the research may follow in the future. Method. First: the AA have proposed a five point definition of this "new problem". Second: they have searched in the on line database the evidence that study this "new problem". Third: the AA have chosen some variables (technology, problem, methodology) with which the evidences founded have been read and each evidence has been screened with those three kind of variables. Forth: the screened variables have been added and commented. Output. A first point is that is possible to identify a second generation of studies on the association/correlation between DTU (Digital Technology Users) and the effects on the brain and the psyche like mental disease. Those studies inquire cohorts of DTU using the golden standard instruments and this has to be a point of no return. The second point is that though DTU population studied is small and not homogeneous, there are evidences about DSM-IV Axis I and II disease. About the problem of dependence/addiction there is a problem to understand. At the moment, with DSM-IV/ICD-10 is possible to make a diagnosis of "dependence" only with "substances". There are some anecdotic evidences that showed a problem of "dependence/addiction" in DTU. Considerations. The AA propose six criteria that the future studies have to be made with. There really is a need to organize international studies following the six criteria proposed.

Key words: Internet, addiction, definition, criteria, DSM-IV 


\section{DEFINICIÓN.}

E n la tabla 1 se proponen los puntos que definen el problema. El punto 1 define la TD (Tecnología Digital) como instrumento de diversas aplicaciones y en continua evolución. El punto 2 define tres posibles modalidades de uso: la lúdica relativa al juego, la profesional relativa al trabajo, y finalmente la utilización relativa a la vida privada como, por ejemplo, las relaciones familiares y amistosas. El punto 3 define la salud, tanto física como mental. El punto 4 define dos posibilidades sustanciales de relacionar entre sí los puntos 1, 2 y 3 . Por "correlación" entendemos un nexo causal, es decir, una relación de causa-efecto entre el uso, la tecnología y la salud. Se entiende, por tanto, que hay al menos una hipótesis respecto al hecho de que el efecto negativo sobre la salud es consecuente al uso de la T D. Por "asociación", en cambio, se considera que no hay ninguna hipótesis de nexo causal entre los puntos 1,2 y 3 , si no simplemente una copresencia frecuente. El punto 5 define las modalidades relativas al uso (2). Se juzga útil, al menos hasta el momento, no incluir el punto 5 en el 2 porque ello permite una mayor claridad.

Tabla 1. Puntos que definen el nuevo problema.

\begin{tabular}{|c|l|l|}
\hline 1 & Variables & \multicolumn{1}{c|}{ Definición } \\
\hline 2 & Tecnología Digital (TD) & $\begin{array}{l}\text { - Internet: navegación hipertextual, líneas de chat, correo } \\
\text { electrónico, juegos on line. } \\
\text { - Playstation. } \\
\text { - Teléfono. }\end{array}$ \\
\hline 3 & Salud (S) & $\begin{array}{l}\text { - Utilización lúdica. } \\
\text { - utilización profesional. } \\
\text { - utilización vida privada. }\end{array}$ \\
\hline 4 & Correlación/asociación (C/A) & $\begin{array}{l}\text { - salud mental: trastornos psíquicos, descuido de las demás } \\
\text { áreas vitales (trabajo, relaciones). } \\
\text { - salud física: posturas, dependencia. }\end{array}$ \\
\hline 5 & Modalidades (M) & $\begin{array}{l}\text { Correlación: la hipótesis de que exista un nexo causal que } \\
\text { relaciona un efecto a una variable que se considera como causa } \\
\text { (ej: hepatitis asociada al VIH). } \\
\text { - Asociación: se consideran asociados dos hechos sin que se } \\
\text { suponga un nexo causal. }\end{array}$ \\
\hline $\begin{array}{l}\text { - tiempo de uso: la definición de un tiempo máximo más allá del } \\
\text { cual podría considerarse constitutivo de un problema, no es aún } \\
\text { posible a no ser en lo que respecta a los problemas posturales. } \\
\text { - modalidades de uso: también en este caso existen indicaciones } \\
\text { de prevención en lo que respecta a la higiene del ambiente y de } \\
\text { la postura. } \\
\text { - elementos asociados (ej: uso de sustancias, prácticas sexuales, } \\
\text { bebidas alcohólicas consumidas durante el uso, etc). }\end{array}$ \\
\hline
\end{tabular}

Variables = los elementos que definen el problema;

Definición = cada variable tiene una definición propia que puede componerse de diversos aspectos.

Del entrelazado de estos cinco elementos distintos resulta un problema de relevancia clínica. Para comprender mejor la tabla $\mathrm{n}^{\circ} 1$ podemos proponer algunas simulaciones. Las Playstation (1) que los niños utilizan para divertirse (2) pueden producir problemas en términos de ansiedad (3) en el momento en que el padre establece un límite; puede darse una correlación (4), es decir, se supone una relación de causa-efecto entre la interrupción del uso y la aparición de la ansiedad, y el problema puede identificarse en términos de tiempo de uso.

Un segundo caso podría ser el de una persona que utilizara mucho las líneas de chat (1) como pasatiempo en su vida privada (2) descuidando otras obligaciones (3) como el trabajo o la familia; puede suponerse aquí 
una relación causal (4) siendo el tiempo (5) el elemento crítico.

Al leer la tabla 1 debe considerarse necesariamente el hecho de que las TD están en continua y rapidísima evolución por lo que las aplicaciones utilizadas actualmente podrían no existir mañana. Además, también es preciso considerar que la cibernética está en continuo crecimiento, ya sea por lo que respecta al número de contratos con los servidores, como por lo que respecta a la familiaridad con el uso. Por último se observa que la aparición de la tecnología UMTS, siglas de Universal Mobile Technology System conllevará necesariamente un significativo salto de calidad en términos de prestación.

\section{METODOLOGÍA BE.}

La metodología BE encuentra su utilización específica en los estudios dirigidos a establecer la eficacia de los tratamientos. Los centros Cochrane son los puntos de referencia esenciales en este ámbito, en el que el trabajador sanitario puede verificar si dicho tratamiento es más o menos eficaz. Los estudios idóeneos para establecer una prueba de eficacia son los RCT, siglas de Randomized Control Study (Control de Estudios Aleatorizados), y en segundo lugar las revisiones sistemáticas y los meta-análisis basados precisamente en los CEA.

La premisa sobre la que se basan los estudios de las pruebas de eficacia, es que exista un trastorno reconocido por la nosografía en vigor en la época presente, ICD-10 y DSM-IV. El objeto de nuestra trabajo es, en cambio, un trastorno aún no reconocido por la nosografía actual. Se trata de indagar la posibilidad de que el uso de la tecnología digital en sus diversas aplicaciones pueda ser "objeto" de dependencia/adicción y que pueda ser causa/cocausa de trastornos psíquicos.

En este caso los estudios útiles pueden ser, por ejemplo, los estudios de cohorte en los que se toman dos grupos de población, uno expuesto a un Factor de Riesgo (FR), y el otro no, verificando cuál de los dos grupos desarrolla un trastorno.

\section{INVESTIGACIÓN DE LAS EVIDENCIAS.}

Se ha procedido, por tanto, a investigar las evidencias en la base de datos disponibles según las variables de la tabla 1. Cada evidencia ha sido también analizada según los cuatro grupos de elementos disgregados a su vez. De cada evidencia se cita el apellido del primer autor y las iniciales del nombre. La tecnología ha sido dividida en videojuegos e Internet. Por el momento no parece oportuno disgregar a su vez Internet, aunque será necesario hacerlo en un futuro, dado que hay demasiados elementos heterogéneos. Por lo que respecta a los problemas es preciso aclarar que se ha elegido este término precisamente por su no especificidad, y que, de cualquier manera, parece un instrumento adecuado en el momento actual. Un término más preciso crearía, a su vez, demasiadas distinciones. La categoría "problemas" ha sido dividida en Adicción, que comprende los comportamientos de dependencia, Sexo, que comprende el complejo mundo de la actividad sexual ejercida por medio del ordenador en Internet. Se usa a menudo el término "cybersex". Se trata de un vasto tema del que solamente podemos intentar proponer algunos elementos: la pornografía digital disponible a través de la conexión con la red sin protección para los menores, el uso de las líneas de chat como vía para contactar con personas con las que organizar encuentros o simplemente estimular el autoerotismo, el intercambio de material pornográfico legal e ilegal y la búsqueda de parejas aptas para requerimientos sexuales particulares. Continuando con el análisis, por Juegos de azar se entiende, en su más amplia acepción, el juego en sus diversas posibilidades, por tanto también en la red. Por Neurofisiología se entiende los estudios, por ejemplo, sobre las habilidades y los tiempos de respuesta a los estímulos. Por Trastornos Psiquiátricos entendemos la presencia de un trastorno psiquiátrico diagnosticado con un instrumento GS (estandarizado y validado). Finalmente, entre los problemas nos encontramos con los forenses relativos a la utilización de las TD y por Delito se entiende el ejercicio de actividades delictivas por medio de las TD en cualquiera de sus diversas aplicaciones.

Pasando a la cuestión metodológica las variables son: GS, siglas de Golden Standard, es decir, instrumentos estandarizados y validados; e Informes Clínicos (IC) significando la descripción, de carácter anecdótico, de algunos casos clínicos; la revisión de la literatura considera la definición de un problema, la presentación de las evidencias científicas y la definición de los criterios con los que se ha efectuado dicho trabajo. Los estudios clínicos aleatorizados son los adecuados para establecer la eficacia de las intervenciones terapéuticas, y en nuestro caso estos estudios son escasamente relevantes ya que no existe una clara categoría diagnóstica de referencia. La epidemiología tiene como objetivo la recogida de datos de las características demográficas y sociales de un determinado problema con el predominio y la incidencia del problema. Los estudios de cohorte están a su vez divididos en los que incluyen el grupo de control y los que no lo contemplan. Por no aplicable (NA), en cambio, se entiende que la evidencia no permite ser interpretada con estas variables. Esto puede depender, o bien de informaciones insuficientes del resumen, o de la ausencia de resumen. 


\section{EL PUNTO DE REFERENCIA}

En 1997,la acreditada publicación American Journal of Psychiatry publicó un artículo, "Internet y el futuro de la Psiquiatría" en el cual los autores, Huang MP y Alessi NE, no mencionaban posibles comportamientos de abuso o síntomas psicopatológicos asociados al uso de la red. En el número siguiente, el $A J P$ incluyó dos breves cartas. En una de ellas, Stein DJ señalaba el hecho de que los autores del artículo citado habían pasado por alto totalmente dos grandes novedades aportadas por Internet en el ámbito psiquiátrico: los comportamientos de abuso y dependencia y las psicoterapias on line. Huang y Alessi respondieron que se trataba de una elección, ya que al no existir literatura que confiriera dignidad científica a estos dos aspectos, opinaban que no debían tratarse.

La postura de Huang-Alessi no ha sido adoptada en ninguna de las aportaciones posteriores a excepción de la presente. Por más que los dos autores no hagan ninguna alusión a la metodología idónea para la elaboración de estudios cualitativamente significativos, ésta es la MBE (Metodología Basada en la Evidencia). Este será el punto de referencia de este trabajo.

Sin que nadie pareciera advertirlo, Koepp MJ et al, publicaron en 1998 una importante estudio en el que se demostraba que utilizando los videojuegos aumentaba la dopamina en la corteza frontal. Por más que el dato sea absolutamente previsible, y por ciertos procedimientos, es obvio que Koepp y su equipo han tenido el mérito de demostrarlo con un estudio de carácter experimental. El hecho de que haya sido demostrado con avanzadas técnicas de neuroimagen confiere un significado importante a la investigación. Existe, en efecto, una correlación neurobiológica que demuestra los efectos en el cerebro de la utilización de los videojuegos.

Más adelante se hará una breve presentación de las contribuciones a la literatura científica que tienen como objeto la tabla $n^{\circ} 1$. Aunque en la práctica clínica los problemas de dependencia/adicción y los trastornos psíquicos se presentan asociados, a menudo definiendo un cuadro de comorbilidad, consideramos oportuno presentar estos dos aspectos por separado.

\section{USO DE LA DTY "DEPENDENCIA".}

Haciendo referencia a la tabla 1, entendemos por dependencia de la TD (1) la utilización (2) de ésta con determinadas modalidades (5) y la generación de comportamientos de dependencia/adicción (4) la hipótesis implícita puede ser de co-rrelación/asociación (3).

Con este propósito podemos identificar dos periodos principales en secuencia temporal que se distin- guen por el paso de artículos de carácter anecdótico (Young KS, 1996; Griffiths M, 1997; Bricolo F et al, 1997) a contribuciones en las que se comienza a investigar sobre muestras numéricamente significativas, utilizando instrumentos GS. Este grupo de estudios se sitúa en la segunda mitad de los años 90 y constituye una excepción el de Brenner V. (1997), porque presenta una investigación sobre una muestra de usuarios de los que analiza el tiempo de conexión. El dato resultante de dichos estudios es que cualquiera que sea el modo en que se describan los problemas del uso de la TD, éstos están asociados, si no correlacionados, con problemas clínicos.

En cambio, a partir de 1999 aparecen estudios en los que se supera el carácter anecdótico y se tiende a los estudios de cohorte. Cantelmi y su grupo realizan aportaciones (Cantelmi T et al, 1999; Talli $\mathrm{M}$ et al, 1999; Del Miglio C et al, 1999) en las que se utilizan instrumentos GS (MMPI-II Hathaway SR, McKinley JC, 1995 y Big five Caparra GV, Barbaranelli C, Borgogni L, 1993) y cuya conclusión es: "Los estudios llevados a cabo hasta ahora, incluido el nuestro, no están en grado de establecer una relación de causaefecto entre la presencia de síntomas y el uso de la red. No obstante, puede destacarse que ciertas características de personalidad contribuyen a determinar una condición de vulnerabilidad, así como una elevada recepción de cementos digitales nocivos. Internet posee, presumiblemente, un potencial psicopatológico, pero sólo en correspondencia a una predisposición individual". Se observan en particular los resultados de las escalas DP (Desviación Psicopática), D (Depresión) del MMPI-II y las escalas EE (Estabilidad Emocional) y CE (Control Emocional) del BFO. "Gracias a la posibilidad de mantener el anonimato, la red puede promover una sustancial disminución de las defensas inhibitorias capaces de facilitar la expresión pulsional. Quienes tiendan a perder el control de la propia "capacidad instintiva" pueden elegir Internet como una especie de válvula de escape a través de la cual canalizar las propias pulsiones de modo socialmente congruente" (Del Miglio C et al, 1999).

Antes de pasar a otro tema vale la pena observar que los estudios de 1999 llevados a cabo en Italia por el grupo de Cantelmi y de Del Miglio, tienen una estructura metodológicamente válida y confirman la presencia de problemáticas clínicas "asociadas" al uso de la TD, excluyendo claramente la "correlación", es decir, una relación de causa-efecto.

\section{USO DE LATDYTRASTORNOS PSÍQUICOS.}

El primer estudio que investigó la relación entre videojuegos y psicopatología es el de McClure RF, Mears FG (1986). Posteriormente se efectuaron otros estudios: uno sobre los aspectos agresivos de meno- 
res (Chambers JH, Ascione FR, 1987); dos sobre la correlación entre epilepsia y videojuegos (De Marco P, Ghersini L, 1985; Masnou P, Nahum-Moscovoci L, 1999); un interesante estudio que investigaba la correlación entre obesidad, TV y videojuegos en los niños mejicanos (Hernández B et al, 1999); y también hacia finales de los años 90 se realizó una primera aportación en la que se menciona la Playstation (Ryan G, 1988). Ninguno de estos estudios ha llamado la atención de los medios de comunicación.

Los estudios que investigan más específicamente el amplio espectro de los Trastornos Psíquicos asociados/correlacionados con el uso de las TD son, una vez más, atribuibles al grupo de Cantelmi T (1999) con la aportación teórica de Carreti V (1999). Indudablemente, a pesar de su carácter anecdótico, se trata de las primeras descripciones de TP asociados al uso de la TD. El cambio metodológico tiene lugar en el 2000, año en el que se publica el trabajo de Saphira NA et al, que presenta una novedad importante. Se utiliza un instrumento GS para evidenciar los Trastornos Psíquicos en Asse $1^{\circ}$ en una muestra de usuarios. En el mismo año, Bricolo F aplica un instrumento GS (SCIDI/ Structured Clinical Interview for Disorder Axix $1^{\circ}$ First MB, el tal, 1994) a dos poblaciones de usuarios profesionales de ordenador/Internet, una población con antecedentes de "enganches" informáticos y de pertenencia al underground, y la otra no. Los usuarios profesionales del primer grupo presentan, de manera homogénea, Trastornos de Personalidad Antisocial y Obsesivo-Compulsivos y Paranoides. El límite del estudio es, ciertamente, el exiguo número (cuatro). Ambos estudios tienen el mérito de utilizar instrumentos GS, Shapira lo suministra a un número bastante significativo, mientras que Bricolo propone un número insuficiente, demasiado pequeño para ser relevante.

Finalmente se hace una consideración con respecto a dos aspectos particulares. La TD, y en particular Internet, con las diversas aplicaciones con las que cuenta en la actualidad, contempla la posibilidad de asumir otras identidades cambiándolas continuamente o, por el contrario, de vivir durante años con una identidad virtual distinta de la real. Un segundo aspecto considera la cuestión relativa a la impulsividad/compulsividad. Se trata de un problema ya difícilmente definible en la psicología/psiquiatría tradicional. En el ámbito del uso de la TD resulta aún más difícil encontrar orientaciones. Estos aspectos deberán ser cuidadosamente investigados a partir de la propuesta de una primera definición de relación de los términos impulsividad/compulsividad e identidad.

\section{EVIDENCIAS.}

La tabla $n^{\circ} 2$ ofrece una visión de conjunto de las evidencias en el ámbito de la correlación entre salud mental y uso de la tecnología digital. Leyendo la tabla de izquierda a derecha resultan algunas de las tipologías principales. 1) Se cita el apellido del primer autor, año de edición del estudio y el país. 2) Tipología de la tecnología digital utilizada, que son esencialmente videojuegos e Internet. 3) Tipología de los problemas investigados que son la adicción, el sexo, los trastornos psíquicos y las actividades de tipo delictivo. 4) Por metodología se entiende el tipo de estudio llevado a cabo y la utilización de instrumentos Golden Standard.

Tabla 2. Parrilla de lectura de las evidencias.

\begin{tabular}{|c|c|c|c|c|c|c|c|c|c|c|c|c|c|c|c|c|c|c|c|c|}
\hline & \multicolumn{3}{|c|}{ EVIDENCIAS } & \multicolumn{2}{|c|}{ Tecnología } & \multicolumn{7}{|c|}{ Problemas } & \multicolumn{8}{|c|}{ Metodología } \\
\hline & Autores & Año & $P$ & V & 1 & A & $S$ & J & $\mathrm{N}$ & TP & $\mathrm{F}$ & D & GS & IC & $A n$ & CEA & E & $\mathrm{CC}$ & C & NA \\
\hline 1 & De Marco P & 1985 & USA & $X$ & & & & & & $X$ & & & & & & & & & & $X$ \\
\hline 2 & McClure RF & 1986 & USA & $x$ & & & & & & $x$ & & & & & & & & & & $x$ \\
\hline 3 & Chambers JH & 1987 & USA & $x$ & & & & & & & & & & & & $x$ & & & & \\
\hline 4 & Clark JE & 1987 & USA & $x$ & & & & & $X$ & & & & & & & & & & & \\
\hline 5 & Bentall RP & 1989 & & & & & & $x$ & & & & & & & & & $x$ & & & $x$ \\
\hline 6 & Griffiths MD. & 1991 & $\mathrm{~GB}$ & $x$ & & & & & & & & & & & & & & & & \\
\hline 7 & Dustman RE & 1992 & USA & $X$ & & & & & $X$ & & & & & & & & & & & $X$ \\
\hline 8 & Young KS & 1996 & USA & & $X$ & & & & & & & & & $X$ & & & & & & \\
\hline 9 & Carr RD & 1996 & USA & $X$ & & & & & & & & & & & & & & & & \\
\hline 10 & Brenner V & 1997 & USA & & $X$ & $X$ & & & & & & & & & & & & & $x$ & \\
\hline 11 & Bricolo F & 1997 & Italia & & $x$ & $x$ & & & & & & & & & & & & & $x$ & \\
\hline 12 & Kofoed L & 1997 & USA & $X$ & & & & & & $X$ & & & $X$ & & & & & $X$ & & $x$ \\
\hline 13 & Cate FH & 1998 & USA & & & & $X$ & & & & $X$ & & & & & & & & & \\
\hline 14 & Strano M & 1998 & Italia & & $x$ & & & & & $x$ & & & & & & & & & $x$ & \\
\hline 15 & Griffiths M & 1998 & $\mathrm{~GB}$ & $x$ & & $X$ & & & $X$ & & & & $X$ & & & & & & & $X$ \\
\hline 16 & Koepp MJ & 1998 & GB & $X$ & & & & & & & & & & & & & & & $X$ & \\
\hline
\end{tabular}




\begin{tabular}{|c|c|c|c|c|c|c|c|c|c|c|c|c|c|c|c|c|c|c|c|c|}
\hline & \multicolumn{3}{|c|}{ EVIDENCIAS } & \multicolumn{2}{|c|}{ Tecnología } & \multicolumn{7}{|c|}{ Problemas } & \multicolumn{8}{|c|}{ Metodología } \\
\hline & Autores & Año & $\mathrm{P}$ & V & I & A & $\mathrm{S}$ & $\mathrm{J}$ & N & TP & $\mathrm{F}$ & D & GS & IC & An & CEA & $\mathrm{E}$ & $\mathrm{CC}$ & $\mathrm{C}$ & NA \\
\hline 17 & Eppright T & 1999 & & & $x$ & & & & & & & & & & $x$ & & & & & \\
\hline 18 & Masnou P & 1999 & Francia & $X$ & & & & & & & & & & & & & & & & $x$ \\
\hline 19 & Hernández B & 1999 & Mexico & $x$ & & & & & & & & & & & & & $x$ & & & $x$ \\
\hline 20 & Cantelmi T & 1999 & Italia & & $x$ & & & & & & & & $x$ & & & & & & $x$ & \\
\hline 21 & Del Miglio & 1999 & Italia & & $x$ & $x$ & & & & & & & $x$ & & & & & & & \\
\hline 22 & Deirmenjian J & 1999 & USA & & $x$ & & & & & & & $x$ & & & & & & & & $x$ \\
\hline 23 & Greenberg JL & 1999 & USA & $x$ & $x$ & & & $x$ & & & & & & & & & & & $x$ & \\
\hline 24 & Bull SS & 2000 & USA & $x$ & & & $x$ & & & & & & & & & & & & $x$ & \\
\hline 25 & Korn DA & 2000 & Canadá & $x$ & & & & & & & & & & & & & $x$ & & & \\
\hline 26 & Oliveira MP & 2000 & Brasil & $x$ & & & & & & & & & $x$ & & & & & & $x$ & \\
\hline 27 & Chau AW & 2000 & Hong Kong & & & & & $x$ & & & & & & & & & & & & \\
\hline 28 & Pugh P & 2000 & GB & & & & & $x$ & & & & & & & & & $x$ & & & \\
\hline 29 & Cantelmi T & 2000 & Italia & & $x$ & $x$ & & & & & & & & & & & & & & \\
\hline 30 & Tisserand IN & 2000 & Francia & & $x$ & $x$ & & & & & & $x$ & & & & & & & & $x$ \\
\hline 31 & Shapira NA & 2000 & USA & & $x$ & & & & & & & & $x$ & & & & & & $x$ & \\
\hline 32 & Ross MW & 2000 & USA & & $x$ & & $x$ & & & & & & & & & & & $x$ & & \\
\hline 33 & Richard J & 2000 & & & $x$ & & & & & $X$ & & & & & & & & & & $x$ \\
\hline 34 & McFarlane M & 2000 & USA & & $x$ & & $x$ & & & & & & & & & & & & $x$ & \\
\hline 35 & Doiron JP & 2001 & & & & & & $x$ & & & & & & & & & & & & $x$ \\
\hline 36 & Sjoberg L & 2001 & Suecia & & & & & $x$ & & & & & & & & & & & $x$ & \\
\hline 37 & Christensen M & 2001 & USA & & $x$ & $x$ & & & & & & & & $x$ & & & & & & $x$ \\
\hline 38 & Goodson P & 2001 & USA & & $x$ & & $x$ & & & & & & & & & & & & & $x$ \\
\hline 39 & Stein DJ & 2001 & S. Africa & & & & & & & & & & & & & & & & & \\
\hline 40 & Dejoie JF & 2001 & Francia & & $x$ & & $x$ & & & & & & & & & & & & & $x$ \\
\hline 41 & Bricolo F & 2001 & Italia & & $x$ & $x$ & & & & & & $x$ & $x$ & & & & & $x$ & & \\
\hline & TOTAL & & & 16 & 19 & 8 & 6 & 6 & 3 & 5 & 1 & 3 & 7 & 2 & 1 & 1 & 4 & 3 & 10 & 11 \\
\hline
\end{tabular}

P = País; V = Videojuegos; I = Internet; A= Adicción; S = Sexo; J = Juegos de azar; N = Neurofisiología; TP = Trastornos Psiquiátricos; F = Forenses; $D$ = Delictivos; GS =Gold Standard (Intrumentos estandarizados y validados); IC =Informe de casos; An = Análisis; $\mathrm{CEA}=$ Control de Estudios Aleatorizados; $\mathrm{E}=$ Epidemiología; $\mathrm{CC}=$ Cohorte con grupo de control; $\mathrm{C}=$ Cohorte; NA = No Aplicable.

De la lectura de la tabla pueden extraerse algunas consideraciones.

Primeramente podemos identificar los trastornos investigados, diferenciándolos de los problemas. Los trastornos son: dependencia/adicción, pedofilias, trastornos disociativos, alteraciones del humor y alteraciones de la personalidad. Los problemas son: delito y suicidio. Los estudios que investiguen estos trastornos deberán ser necesariamente repetidos/falsificados teniendo presente la dificultad de la homogeneidad de la muestra investigada en las distintas culturas.

Si en un principio los estudios se concentraron sobre los videojuegos como aplicación tecnológica (entonces analógica), ahora, sin duda, la atención se polariza en Internet. Ya hemos señalado anteriormente el problema de la heterogeneidad en esta categoría y la necesidad de llevar a cabo investigaciones específicas para las aplicaciones.

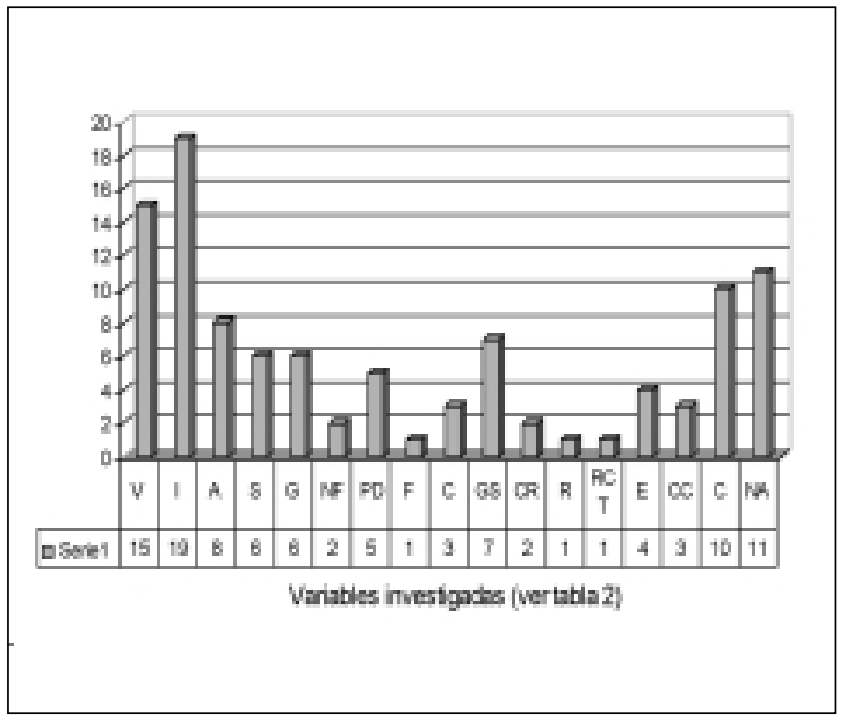

Figura 1. Representación gráfica de la Parrilla. 
Destacamos la presencia de estudios que utilizan instrumentos GS y el hecho de que el conjunto de los estudios comprendidos en la categoría "Cohorte" sean 13. Finalmente también se observa que, como resulta de la categoría NA = 11, no siempre los resúmenes ofrecen suficiente información para falsificar el estudio en términos BE.

\section{CENTROS DE ESTUDIO.}

Teniendo presente la tabla $n^{\circ} 2$ se ha procedido a identificar a aquellos autores que han elaborado estudios metodológicamente correctos y que pueden, por tanto, servir de referencia para informaciones y eventuales peticiones de ayuda.

Tabla 3. Centros de estudio de referencia

\begin{tabular}{|c|c|c|}
\hline \multicolumn{3}{|c|}{ Centros de estudio de referencia } \\
\hline Autores & E-mail & Institución \\
\hline Bricolo F. & fbricolo@dronet.org & $\begin{array}{l}\text { Dipartimento delle Dipendenze (Addiction Department) } \\
\text { Via Germania } 20 \text { I-37135- Verona Italy }\end{array}$ \\
\hline Cantelmi T. & $\begin{array}{l}\text { tcantelmi@getmet.it } \\
\text { (psychoinside@tiscalinet.it) }\end{array}$ & $\begin{array}{l}\text { Centro di Psichiatria e Psicologia Clinica, Roma } \\
\text { (http://www.psychoinside.it/i) }\end{array}$ \\
\hline Strano M. & strano@skynet.it & $\begin{array}{l}\text { Istituto Universitario di Ricerca Criminologia, Via } \\
\text { Circonvallazione Ostiense 169, interno 2, ROMA. }\end{array}$ \\
\hline Deirmenjian JM & jdeirme@earthlink.net & $\begin{array}{l}\text { Dept. of Psychiatry, Case Western Reserve University } \\
\text { School of Medicine, Cleveland, OH, USA. }\end{array}$ \\
\hline Pratarelli ME & marcp@okway.okstate.edu & $\begin{array}{l}\text { Department of Psychology, Oklahoma State University, } \\
\text { Stillwater 74078, USA. }\end{array}$ \\
\hline Shapira NA & shapira@psych.med.ufl.edu & $\begin{array}{l}\text { Department of Psychiatry, University of Florida, P.O.Box } \\
\text { 100256, Gainesville, FL 32610-0256, USA. }\end{array}$ \\
\hline Chau AW & awlchau@hkucc.hku.hk & $\begin{array}{l}\text { Department of Psychology, The University of } \\
\text { Hong Kong. }\end{array}$ \\
\hline Sjoberg L & pls@hhs.se & $\begin{array}{l}\text { Stockholm School of Economics, Centre for Risk } \\
\text { Research }\end{array}$ \\
\hline McFarlane M & mmcfarlane@cdc.gov & $\begin{array}{l}\text { Division of Sexuality Transmitted Disease Prevention } \\
1600 \text { Clifton Rd, MS E-44, Atlanta, GA } 30333\end{array}$ \\
\hline Richard J & Jon.Richard@state.co.us & $\begin{array}{l}\text { Colorado Mental Health Institute at Fort Logan, } 3520 \\
\text { West Oxford Ave., Denver, CO } 80236 .\end{array}$ \\
\hline
\end{tabular}

En el contenido de la tabla 3 observamos que, si bien los autores han realizado estudios sobre temáticas no encuadrables en los puntos de la tabla $n^{\circ} 1$, no siempre efectúan proyectos para la llevar a la práctica clínica. Se proponen, de todos modos, como puntos de referencia.

Desde este punto de vista son las realidades locales las que acogen la exigencia de organizar proyectos, tanto en lo que respecta a la encuesta como a la terapia. La región del Veneto (Italia) ha incluido entre las "Normas de intervención del sector de las dependencias" un nuevo apartado en el que se solicitan proyectos en este sentido, reconociéndolos, de esta manera, como competencia de los Departamentos de Dependencias.

\section{CONCLUSIONES.}

La utilización de instrumentos GS en los últimos estudios de los años 90 y en los primeros del 2000, representa verdaderamente una novedad significativa en términos cualitativos. El la tabla $n^{\circ} 4$ se enumeran y describen los cinco puntos considerados fundamentales para las investigaciones de calidad sobre estas nuevas temáticas. 
Tabla 4. Indicaciones para la investigación futura.

\begin{tabular}{|cc|l|}
\hline 1 & Definición. & $\begin{array}{l}\text { Indicaciones para la investigación futura. } \\
\text { Verificar/falsificar lo propuesto en la tabla n } 1 \text { sobre el problema de investigar } \\
\text { particularmente lo que se refiere al punto nº 3. }\end{array}$ \\
\hline 2 & Método. & $\begin{array}{l}\text { En la fase actual, al no estar clara la diagnosis de salida, no parece que sean } \\
\text { útiles lo estudios destinados a medir la eficacia de las terapias. Por tanto, parece } \\
\text { importante efectuar estudios de cohorte y, en particular, estudios de cohorte que } \\
\text { incluyan grupo de control (por ejemplo, un grupo de población de "Uso excesivo" } \\
\text { frente a otro grupo de "Uso moderado"). }\end{array}$ \\
\hline 3 & Número. & $\begin{array}{l}\text { Las indicaciones de la metodología BE consideran que para definir un problema } \\
\text { es necesario investigar grupos de población numéricamente significativos. }\end{array}$ \\
\hline 4 & Instrumentos G S. & $\begin{array}{l}\text { Utilización de instrumentos "golden standard", es decir, instrumentos } \\
\text { estandardizados y validados. }\end{array}$ \\
\hline 5 & "Problemas" & $\begin{array}{l}\text { Problemas: tutela de menores, delincuencia, seguridad, dependencia/adicción, } \\
\text { trastornos psíquicos, legalidad, ética. Cada una de estas áreas se indagan por } \\
\text { separado con métodos e instrumentos adecuados. }\end{array}$ \\
\hline 6 & Globalización. & $\begin{array}{l}\text { La introducción de este concepto lleva a modificar algunos puntos de referencia } \\
\text { como los conceptos de "límite", "distancia", "identidad", "comunicación" y "cultura", } \\
\text { sirviendo así de orientación también por lo que respecta al ámbito clínico. Este } \\
\text { aspecto ha de tenerse en consideración. }\end{array}$ \\
\hline
\end{tabular}

La investigación debe orientarse hacia estudios de cohorte con grupo de control (2) sobre muestras numéricamente significativas (3), utilizando instrumentos GS (4), investigando problemas específicos (5) teniendo en cuenta la necesidad de redefinir la variable "cultura" en la época de la globalización (6). Además, estos estudios deben servir para falsificar (1) la definición de la tabla 1.

Por lo que respecta a la posibilidad de que este nuevo problema se convierta en una "nueva categoría" dentro de los sistemas nosográficos oficiales (DSM e ICD), hay que tener en cuenta que el camino que lleva a la formulación de una categoría o de un trastorno tiene unas etapas preestablecidas por la Asociación de Psiquiatría Americana y la Organización Mundial de la Salud. En este complejo procedimiento se efectúan estudios según las indicaciones de la tabla 4 y se desaconsejan las propuestas que no estén basadas en investigaciones acordes con los criterios anteriormente expuestos.

Respecto a la posibilidad de que en los sistemas nosográficos futuros puedan introducirse como categoría aparte los "Trastornos correlacionados con el uso de la DT" o que, por el contrario, puedan incluirse nuevos trastornos en categorías ya existentes; en el momento actual nos parece más adecuada la segunda posibilidad.

\section{BIBLIOGRAFÍA}

1. American Psychiatric Association. Diagnostical and Statistical Manual of Mental Disorders DSM-IV, Masson, 1994

2. Bentall RP, Fisher D, Kelly V, Bromley E, Hawksworth K. The use of arcade gambling machines: demographic characteristics of users and patterns of use. $\mathrm{Br} \mathrm{J}$ Addict 1989 May;84(5):555-62

3. Brenner V, Psychology of computer use: XLVII. Parameters of Internet use, abuse and addiction: the first 90 days of the Internet Usage Survey. Psychol Rep, 80:879-82, 1997 Jun

4. Bricolo F, Marconi PL, Conte GL, di Giannantonio M, De Risio S, Internet Addiction Disorder: una nuova dipendenza? Studio di un campione di giovani utenti. SIP, Bollettino Scientifico e d'informazione, n. 1-2, anno IV, marzo-luglio 1997, pp. 38-44;

5. Bricolo F, "Correlare" la salute mentale all'uso della tecnologia digitale. Revisione critica della letteratura. Personalità e Dipendenze, Fasc. 1 2001, pp.11-16

6. Brown CS, Kent TA, Blood platelet uptake of serotonin inepisodic aggression. Psychiatry Research, 1989, 27, 512

7. Bull SS, McFarlane M, Soliciting sex on the Internet: what are the risks for sexually transmitted diseases and HIV? Sex Transm Dis 2000 Oct;27(9):545-50

8. Cantelmi T, Talli M, D’Andrea A, Del Miglio C, "La mente in Internet", Piccin Editore, Padova 2000 
9. Cantelmi T, Talli M, D’Andrea A, Gasbarri A, "Internet Related Psychopathology: recenti acquisizioni", Giornale Italiano di Medicina Militare, 1999

10. Cantelmi T, De Marco M, Talli M, Del Miglio C, Internet Related Psychopathology: aspetti clinici e recenti acquisizioni, in: Attualità in psicologia, Vol. 15, n. 2 pp. 194-5, 2000

11. Caparra GV, Barbaranelli C, Borgogni L, BFO Big Five Questionnarie, OS, Firenze, 1993

12. Caretti $V$, "Psicodinamica della Trance Dissociativa da videoterminale", in Cantelmi T. et al, "La mente in Internet", Piccin Editore, Padova, 2000

13. Cate FH, Cybersex: regulating sexually explicit expression on the Internet. Behav Sci Law 1996 Spring; 14(2):145-66

14. Chambers JH; Ascione FR, The effects of prosocial and aggressive videogames on children's donating and helping. J Genet Psychol, 148:499-505, 1987 Dec

15. Chau AW, Phillips JG, Von Baggo KL. Departures from sensible play in computer blackjack. J Gen Psychol 2000 Oct;127(4):426-38

16. Christensen $\mathrm{MH}$, Orzack $\mathrm{MH}$, Babington LM, Patsoaughter CA. Computer addiction. When monitor becomes control center. J Psychosoc Nurs Ment Health Serv 2001 Mar;39(3):40-7

17. Clark JE, Lanphear AK, Riddick CC, The effects of videogame playing on the response selection processing of elderly adults. J Gerontol, 42:82-5, 1987 Jan

18. Del Miglio C, Cantelmi T, Talli M, Artelli F, Cavolina P, "Fenomeni psicopatologici Internet-correlati: ricerca sperimentale italiana", in Cantelmi T. et al, "La mente in Internet", Piccin Editore, Padova, 1999 p. 92-93

19. Dejoie JF, [Internet addiction: a different kind of addiction?]. Rev Med Liege 2001 Jul;56(7):523-30

20. De Marco P, Ghersini L, Videogames and epilepsy. Dev Med Child Neurol, 15:519-21, 1985 Aug

21. Deirmenjian JM. Stalking in cyberspace. J Am Acad Psychiatry Law 1999;27(3):407-13

22. Deirmenjian JM. Hate crimes on the internet. J Forensic Sci 2000 Sep;45(5):1020-2

23. Doiron JP, Mazer DB, Gambling with video lottery terminals. Qual Health Res 2001 Sep;11(5):631-46

24. Doiron JP, Nicki RM, Epidemiology of problem gambling in Prince Edward Island: a Canadian microcosm? Can J Psychiatry 2001 Jun;46(5):413-7

25. Dustman RE, Emmerson RY, Steinhaus LA, Shearer DE, Dustman TJ, The effects of videogame playing on neuropsychological performance of elderly individuals. J Gerontol, 47:P168-71, 1992 May

26. Eppright $T$, Allwood $M$, Stern $B$, Theiss $T$, Internet addiction: a new type of addiction?, Mo Med, 96(4):13361999 Apr;

27. First MB, Spitzer RL, Gibbon M, Williams JBW, Benjamin L,Structured Clinical Interview for DSM-IV Axis II Personalità Disorders (SCID-II) (version 2.0), Biometric Research Department, New York State Psychiatrisc Institute, 722 West 168th Street, NY, NY 10032, 1994; onde evitare malitesi va ribadito che a tutt'oggi non è disponibile una traduzione italiana validata della SCID-II. Perciò tutti coloro che applicano lo SCID-II in italiano fanno uso di traduzioni non ufficiali. Le Organizzazioni Speciali di Firenze pubblicheranno l'edizione tra qualche anno.

28. Galimberti Umberto, Psiche e teche L'uomo nell'età della tecnica, Feltrinelli, 1999

29. Goodson P, McCormick D, Evans A. Searching for sexually explicit materials on the Internet: an exploratory study of college students' behavior and attitudes. Arch Sex Behav 2001 Apr;30(2):101-18

30. Greenberg JL, Lewis SE, Dodd DK. Overlapping addictions and self-esteem among college men and women. Addict Behav 1999 Jul-Aug;24(4):565-71

31. Griffiths M, Psychology of computer use: XLIII. Some comments on 'addictive use of the Internet' by Young [comment], Psychol Rep, 80(1):81-2 1997 Feb

32. Griffiths MD, Hunt N, Dependence on computer games by adolescents. Psychol Rep 1998 Apr;82(2):475-80

33. Griffiths MD, Amusement machine playing in childhood and adolescence: a comparative analysis of video games and fruit machines. J Adolesc 1991 Mar;14(1):53-73

34. Hathaway SR, McKinley JC, MMPI-2 Minnesota Multiphasic Personalità Inventory - 2, OS, Firenze, 1995

35. Hernández B, Gortmaker SL, Colditz GA, Peterson KE, Laird NM, Parra-Cabrera S, Association of obesity with physical activity, television programs and other forms of video viewing among children in Mexico city. Int J Obes Relat Metab Disord, 23:845-54, 1999 Aug

36. Huang MP, Alessi NE, The Internet and the future of psychiatry, Am J Psychiatry, 153:861-9, 1996 Jul; Stein DJ, 1997

37. Korn DA, Expansion of gambling in Canada: implications for health and social policy. CMAJ 2000 Jul 11;163(1): 61-4

38. Koepp MJ, Gunn RN, Lawrence AD, Cunningham VJ, Dagher A, Jones T, Brooks DJ, Bench CJ, Grasby PM. Evidence for striatal dopamine release during a video game. Nature 1998 May 21;393(6682):266-8

39. Kofoed L, Morgan TJ, Buchkoski J, Carr R, Dissociative experiences scale and MMPI-2 scores in video poker gamblers, other gamblers, and alcoholic controls. J Nerv Ment Dis 1997 Jan;185(1):58-60

40. La Barbera D, La Rete che connette, la Rete che cattura: metafore della "esperienza" Internet. in Cantelmi T. et al, "La mente in Internet", Piccin Editore, Padova, 1999

41. Levy S, Cybercultura Gli susi sociali delle nuove tecnologie, Feltrinelli, 1999

42. McClure RF, Mears FG, Videogame playing and psychopathology. Psychol Rep, 59:59-62, 1986 Aug.

43. Masnou P, Nahum-Moscovoci L, Epilepsy and videogame: which physiopathological mechanisms to expect? Rev Neurol (Paris), 155:292-5, 1999 Apr

44. Melis M, Diana M, Gessa GL, I cannabinoidi attivano i neuroni dopaminergici del sistema mesolimbicocorticale, in: Janiri L, Bricolo F, Conte GL, Cannabinoidi: biologia e clinica, SEU, 1998, 
45. Oliveira MP, Silva MT, Pathological and nonpathological gamblers: a survey in gambling settings. Subst Use Misuse 2000 Sep;35(11):1573-83

46. Pratarelli ME, Browne BL, Johnson K, The bits and bytes of computer/Internet addiction: a factor analytic approach. Behav Res Methods Instrum Comput, 31 (2): 305-14 May 1999

47. Young KS, Psychology of computer use: XL. Addictive use of the Internet: a case that breaks the stereotype [see comments], Psychol Rep, 79(3 Pt 1):899-902 1996 Dec

48. Young K.S. "Caught in the Net" John Wiley \& Sons, New York, 1998

49. Young K.S. "Presi nella rete", introduzione di Tonino Cantelmi, 2000

50. Pugh P, Webley P. Adolescent participation in the U.K. national lottery games. J Adolesc 2000 Feb;23(1):1-11

51. Richard J, Werth JL Jr, Rogers JR. Rational and assisted suicidal communication on the Internet: case example and discussion of ethical and practice issues. Ethics Behav 2000;10(3):215-38

52. Riddick CC, Drogin EB, Spector SG, The impact of videogame play on the emotional states of senior center participants. Gerontologist, 155:425-7, 1987 Aug

53. Roy $A$, Linnoila $M$, Suicidal behavior, impulsiveness and serotonin. Acta Pychiatrica Scandinava, 1988, 78, 529535

54. Ross MW, Tikkanen R, Mansson SA. Differences between Internet samples and conventional samples of men who have sex with men: implications for research and HIV interventions. Soc Sci Med 2000 Sep;51(5): 749-58

55. Samoilovich S, Riccitelli C, Schiel A, Siedi A, Attitude of schizophrenics to computer videogames. Psychopathology, 25:117-9, 1992

56. Shapira NA, Goldsmith TD, Keck Jr, Khosla UM, McElroy SL, Psychiatric features of individuals with problematic internet use. J Affect Disord, 57(1-3): 267-72 2000 Jan-Mar

57. Sjoberg L, Fromm J, Information Technology Risks as Seen by the Public. Risk Analysis, Vol. 21, N. 3, 2001

58. Stein DJ, Internet addiction, Internet psychotherapy [letter; comment] Am J Psychiatry, 154(6):890 1997 Jun

59. Stein DJ, Black DW, Shapira NA, Spitzer RL. Hypersexual disorder and preoccupation with internet pornography. Am J Psychiatry 2001 Oct;158(10):1590-4

60. Strano M, Pedofilia e internet: quali rischi per i minori, BYTE, ottobre 1998;

61. Strano M., pedofilia e telematica: la ricerca criminologica sul web in Cantelmi T., Del Miglio C, Talli M, D'Andrea A, La mente in internet, Piccin, Padova, 1999;

62. Talli M, D'Andrea A, Cantelmi T, Strumenti clinici di valutazione, in Cantelmi T. et al, "La mente in Internet", Piccin Editore, Padova, 1999 pp. 115-124

63. Tisserand IN, [New risks of addiction for new populations: the example of hackers], Ann Med Interne (Paris) 2000 Oct;151 Suppl B:B49-52 